\title{
Unknown Intent Detection Using Multi-Objective Optimization on Deep Learning Classifiers
}

\author{
Prerna Prem $^{1}$, Zishan Ahmad ${ }^{1}$, Asif Ekbal ${ }^{1}$ \\ Shubhashis Sengupta ${ }^{2}$, Sakshi C. Jain ${ }^{2}$, Roshni Ramnani ${ }^{2}$ \\ ${ }^{1}$ Department of Computer Science and Engineering, Indian Institute of Technology Patna \\ ${ }^{2}$ Accenture Technology Lab, Bangalore \\ prernaprem21@gmail.com 1821cs18@itp.ac.in \\ asif.ekbal@gmail.com shubhashis.sengupta@accenture.com \\ sakshi.c.jain@accenture.com roshni.r.ramnani@accenture.com
}

\begin{abstract}
Modelling and understanding dialogues in a conversation depends on identifying the user intent from the given text. Unknown or new intent detection is a critical task, as in a realistic scenario a user intent may frequently change over time and divert even to an intent previously not encountered. This task of separating the unknown intent samples from known intents one is challenging as the unknown user intent can range from intents similar to the predefined intents to something completely different. Prior research on intent discovery often consider it as a classification task where an unknown intent can belong to a predefined set of known intent classes. In this paper we tackle the problem of detecting a completely unknown intent without any prior hints about the kind of classes belonging to unknown intents. We propose an effective post-processing method using multi-objective optimization to tune an existing neural network based intent classifier and make it capable of detecting unknown intents. We perform experiments using existing state-of-the-art intent classifiers and use our method on top of them for unknown intent detection. Our experiments across different domains and real-world datasets show that our method yields significant improvements compared with the state-of-the-art methods for unknown intent detection.
\end{abstract}

\section{Introduction}

Detecting whether an intent is unknown or new in a dialogue system has become an important task for improving customer satisfaction. Since user intent may frequently change over time in many realistic scenarios, unknown (new) intent detection has become a crucial problem in conversational artificial intelligence (CAI). This can ultimately help enhance system interaction with the customer. This task is challenging since there is no prior knowl- edge of the type or the exact numbers of unknown intents that would be encountered in the future.

We model unknown intent detection as an $(\mathrm{m}+1)$ class classification task as suggested by (Shu et al., 2017; Lin and Xu, 2019; Zhang et al., 2020) and consider unknown classes as the $(\mathrm{m}+1)^{\text {th }}$ class. We aim to identify the known intent samples accurately, while at the same time we focus on determining the unknown intent samples. This has to be done without any prior knowledge about the kind of unknown intents. In order to solve this problem, researchers have proposed deep neural networks like OpenMax (Bendale and Boult, 2016), which fits Weibull distribution to the outputs of the penultimate layer. Another system MSP (Hendrycks and Gimpel, 2016) calculates the softmax probability of known samples and discards the unknown samples with lower confidence. In our approach, we attempt to solve the problem of unknown intent detection with added constraints such as not having any prior knowledge of a finite set of intents.

The main contributions of this paper are:

1. We propose an efficient method for unknown intent detection that post-processes using multi-objective optimization (nondeterministic genetic algorithm-NSGA2) by optimising two objectives i.e. recall and precision in order to obtain the optimal thresholds for each intent class.

2. Our proposed weight fine-tuning approach is model-agnostic, i.e. it can be applied on top of any deep neural network model.

The rest of the paper is organized as follows. In Section 2, we present the literature survey of previous work done on this topic. In Section 3 we elaborately describe the proposed methodology. In Section 4 , we discuss the experimental setup and the datasets used in our experiments. In Section 
5 , we analyse the results of detecting the unknown intents. Finally, Section 6 concludes the paper with future work that can be explored in this field.

\section{Related Works}

Intent detection is a much explored area in dialogue systems with a broad spectrum of literature available (Min et al., 2020; Qin et al., 2020; Zhang et al., 2018; Niu et al., 2019; Qin et al., 2019). Most of these works are based on closed world classification that does not consider any open intent. (Srivastava et al., 2018) proposed a zero-shot learning (ZSL) for intent detection. However, ZSL is different from our task as it only contains finite known set of classes during testing. (Kim and Kim, 2018) tried to optimise the intent classifier together with an out-of-domain detector, which was trained using out-of-domain samples. The generative method proposed by (Yu et al., 2017) used adversarial learning to generate positive and negative examples from known classes but the method did not work well in the discrete data space like text. (Ryu et al., 2018) proposed generative adversarial network (GAN) to train on the ID samples and use the discriminator to detect the out-of-domain samples. (Nalisnick et al., 2018; Mundt et al., 2019) showed that deep generative models fail to capture the high-level semantics on real world data. (Jain et al., 2014) fit the probability distributions to statistical Extreme Value Theory (EVT) using a Weibull-calibrated multi-class support vector machine (SVM) to detect the unnormalized posterior probability of inclusion for open set problems. ODIN (Liang et al., 2017) enlarged the differences between known and unknown samples by using temperature scaling and input pre-processing but all the above method need negative samples for selecting the decision boundary or probability threshold. DOC (Shu et al., 2017), instead of using Softmax as the final output layer, built a multi-class classifier with a 1-vs-rest final layer which contains a sigmoid function for each seen class to reduce the open space risk.

Zero-shot intent classification aims to generalize knowledge and concepts learned from the seen intents to recognize unseen intents. Early methods (Ferreira et al., 2015a,b) explored the relationship between seen and unseen intents by introducing external resources such as manually defined attributes or label ontologies, but they are usually expensive to obtain. To deal with this, some methods (Chen et al., 2016; Kumar et al., 2017) map the utterances and intent labels to an embedding space and then model their relations in the same space. IntentCapsNet-ZS (Xia et al., 2018) extends capsule networks (Sabour et al., 2017) for zero-shot intent classification by transferring the prediction vectors from seen classes to unseen classes. ReCapsNet (Liu et al., 2019) shows that IntentCapsNetZS hardly recognizes utterances from unseen intents in the generalized zero-shot classification scenario, and proposes to solve this issue by transferring the transformation matrices from the seen to unseen intents. These approaches also need unknown intent embedding for classifying these instances. Our work do not require the assumption of that classes belong to a closed word. We don't need the unseen intent samples to get the deep learning classifier to detect unknown intents as well.

\section{Methodology}

We train two different deep learning model for intent classification and use our post-processing steps on top of these to obtain optimal results. The pipeline of the system processes is shown in Figure 1. We describe the models along with our novel post-processing steps in this section.

\subsection{Models}

\subsubsection{Bi-LSTM}

We train Bi-directional Long Short Term Memory (Bi-LSTM) to obtain the prediction scores and use these scores to obtain the optimal thresholds for each known intent class using different threshold tuning methods as discussed in 3.3. Given an utterance with maximum word sequence length $l$, we transform a sequence of input words $w_{1: l}$ into mdimensional word embedding $v_{1: l}$, which is used by forward and backward LSTM to produce feature representations $\mathrm{x}$ :

$$
\begin{gathered}
\overrightarrow{x_{t}}=\operatorname{LSTM}\left(v_{t}, \vec{c}_{t-1}\right) \\
\overrightarrow{x_{t}}=\operatorname{LSTM}\left(v_{t}, \vec{c}_{t-1}\right) \\
x=\left[\overrightarrow{x_{l}}: \overrightarrow{x_{1}}\right]
\end{gathered}
$$

where $v_{t}$ denotes the word embedding of input at time step t. $\overrightarrow{x_{t}}$ and $\overrightarrow{x_{t}}$ are the output vector of forward and backward LSTM, respectively. $\overrightarrow{c_{t}}$ and $\overrightarrow{c_{t}}$ are the cell state vectors of forward and backward LSTM, respectively. We concatenate the last output vector of forward LSTM $\overrightarrow{x_{l}}$ and the first output vector of backward LSTM $\overrightarrow{x_{1}}$ into $\mathrm{x}$ as the sentence 


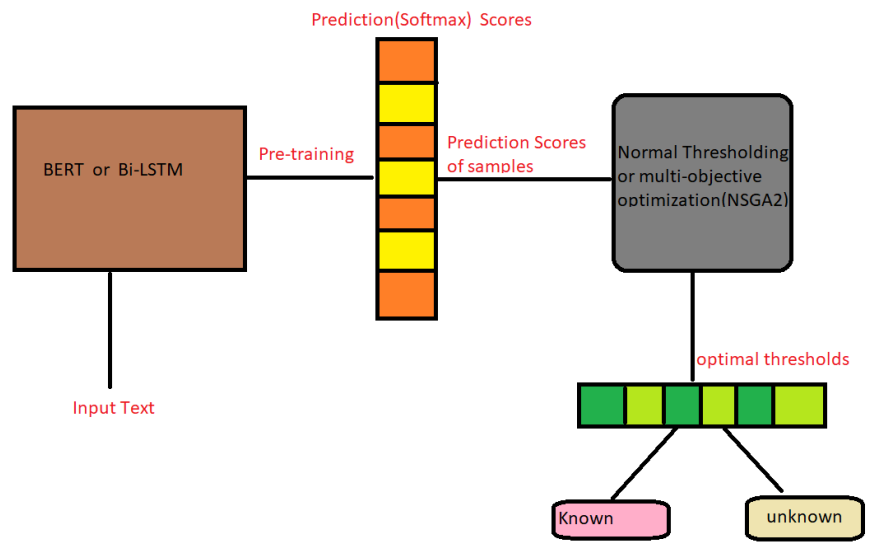

Figure 1: The system architecture consisting of two parts (i). BERT or Bi-LSTM model for softmax score prediction and (ii). Normal Thresholding or NSGA2 for tuning the thresholds of class scores

representation. It captures high-level semantic concepts learned by the model. The representation $\mathrm{x}$ is then fed to an $n$ neuron feed forward layer where $n$ is the number of known intent classes in the dataset. The $n$ dimensional representation obtained is converted to probability distribution by using a 'Softmax' function.

\subsubsection{BERT}

We fine tune the pre-trained Bi-directional Encoder Representation from Transformer (BERT) model to get the 'softmax' classification scores of the input samples. Given $\mathrm{i}^{\text {th }}$ input sentence $s_{i}$ we append a $[C L S]$ token at the beginning of the sentence. We obtain the token embeddings of the sequence $[C L S, T 1, \ldots, T N] \in R^{(N+1) * H}$ from the last hidden layer of BERT. Here the $[C L S]$ vector representation is used for text classification, $N$ is the sequence length and $H$ is the hidden layer size. We calculate the prediction scores by applying 'Softmax' function to the last layer output $\left(\operatorname{logits}\left(x_{i}\right)\right)$ of the trained BERT model.

\subsection{Pre-Training}

After training the intent classification model, for each input we obtain the 'Softmax' scores w.r.t each class at the output layer. We need to set a thresholds for these scores, above which the input sample is classified to the respective class. Since we do not use have any separate class for unknown intent, we train our model on a subset of the classes in the dataset, holding out the rest to be classified as unknown during testing. In order to reflect the effectiveness of the learned optimal thresholds we use cross-entropy loss $L_{s}$ to train our both the base models.

$$
L_{s}=\frac{-1}{N} \sum_{i=1}^{N} y_{i} \log \left(\hat{y}_{i}\right)
$$

where $N$ is total number of training samples $y_{i}$ is true label and $\hat{y}_{i}$ is predicted label. Then, we use the pre-trained model to obtain the prediction scores of the input samples. These scores are used further for threshold tuning of each known intent class.

\subsection{Finding Optimal Threshold for Each Known Intent Class}

To obtain the prediction scores corresponding to each sample we pass the training data samples to the pre-trained classifiers. After getting the prediction scores we apply two different techniques to obtain the optimal threshold for each known intent class, viz. normal thresholding, and multi-objective optimization.

\subsubsection{Normal Thresholding}

In this method, first the input text containing the training data samples is fed to the deep learning classifier to get the prediction scores corresponding to each samples. These prediction scores $(P S)$ and the list of thresholds $(T)$ ranging from 0.1 to 0.9 increasing by 0.1 in each step is used to calculate the correct classification matrix $(C C M)$ and the mis-classification matrix $(M C M)$.

The set of prediction scores is a matrix of $N \times M$ where $N$ is the total number of training samples and $M$ is the number of known intent classes. This 


\begin{tabular}{|c|c|c|c|c|}
\hline Datasets & Classes (intents) & \#Training & \#Validation & \#Test \\
\hline Bank_catridge & 14 & 1020 & 120 & 240 \\
\hline Banking & 77 & 9003 & 1000 & 3080 \\
\hline SNIPS & 7 & 9234 & 1020 & 5060 \\
\hline
\end{tabular}

Table 1: Statistics of the dataset being used in our experiment.

\begin{tabular}{|c|l|}
\hline Datasets & Few examples of intents \\
\hline \multirow{3}{*}{ Bank_catridge } & $\begin{array}{l}\text { ['Bal_Inquiry', 'Card_Activation', 'card_declined', } \\
\text { 'cheque_book_Req', 'credit_query', 'direct_deposit', } \\
\text { 'freeze_account', 'inter_transfer', 'mortgage_processing', } \\
\text { 'replacement_card_duration', 'report_fraud', } \\
\text { 'report_lost_card', 'update_so_dd'] }\end{array}$ \\
\hline Banking & $\begin{array}{l}\text { ['transfer_timing', 'order_physical_card', 'card_acceptance', } \\
\text { 'balance_not_updated_after_bank_transfer','card_swallowed', } \\
\text { 'top_up_by_bank_transfer_charge', 'card_delivery_estimate', } \\
\text { 'transfer_not_received_by_recipient'] }\end{array}$ \\
\hline SNIPS-NLU & ['music','playlist', 'book', 'restaurant', 'search', 'wether'] \\
\hline
\end{tabular}

Table 2: Few intents present in each of the dataset.

set of prediction scores and the list of thresholds containing $K$ threshold values is used to calculate correct classification matrix $(C C M)$ and the missclassification matrix $(M C M)$.

Let $C(X)$ be the output class, $Y$ the ground truth class, and (.) the enumeration function, the standard definition for correctly classified sample (or true positives) rate of an intent class $i$ is given in Equation 1:

$$
C C_{i}=\frac{(C(X)=i A N D Y=i)}{Y=i}
$$

We can also write the standard definition of misclassified sample rate (or false negatives) of an intent class $i$ as given by Equation 2:

$$
M C_{i}=\frac{(C(X) \neq i A N D Y=i)}{Y=i}
$$

The correct classification rate (CC) and misclassification (MC) rate of an intent $i$ can be extended by introducing the thresholds $\tau_{i}$ and by adding the unsure classification (UC) rate, for each intent as shown in Equation 3, 4 and 5.

$$
\begin{gathered}
C C_{i}\left(\tau_{i}\right)=\frac{\left(C(X)=i A N D S(X)>\tau_{i}\right) A N D(Y=i)}{Y=i} \\
M C_{i}\left(\tau_{i}\right)=\frac{\left.(X) \neq i A N D S(X)>\tau_{i}\right) A N D(Y=i)}{Y=i} \\
U C_{i}\left(\tau_{i}\right)=\frac{((C(X)=i) \text { or }(C(X) \neq i) A N D}{\left(S(X)<\tau_{i}\right) A N D(Y=i)} \\
Y=i
\end{gathered}
$$

For each intent we have:

$$
C C_{i}(\tau)+M C_{i}(\tau)+U C_{i}(\tau)=1
$$

$C C M$ is a matrix of $K \times M$ dimension containing the correct classification rate of each intent class corresponding to each threshold in the threshold list i.e each entry $C C_{i j}$ is calculated using equation 6.

$C C_{i j}=\sum_{i=1}^{N} \frac{\left(C(X)=i A N D S(X)>\tau_{j}\right) A N D(Y=i)}{Y=i}$

$M C M$ is a matrix of $K \times M$ dimension containing the mis-classification rate of each intent class corresponding to each threshold in the threshold list i.e each entry $M C_{i j}$ is calculated using equation 7 .

$M C_{i j}=\sum_{i=1}^{N} \frac{\left(C(X) \neq i A N D S(X)>\tau_{j}\right) A N D(Y=i)}{Y=i}$

After obtaining these two matrices, we obtain optimal $\tau_{j}$ for each known intent class by the following technique. We keep the best correct classification rate while reducing the mis-classification rate. For this, we use two steps. First, we determine the threshold(s) $\tau$ which maximizes $C C_{i}(\tau)$. Since several thresholds could reach this maximum, we obtain a set of threshold(s) $S e g_{1}$. Then, we selected the threshold with the lower $M C_{i}(\tau)$. This can be mathematically written as:

$$
\begin{gathered}
s=\operatorname{argmax}_{\tau}\left(C C_{i}(\tau)\right) \\
\tau_{i}=\operatorname{argmin}_{\tau^{\prime} \epsilon s}\left(M C_{i}\left(\tau^{\prime}\right)\right)
\end{gathered}
$$

\subsubsection{Multi-Objective Optimization (NSGA2)}

To get the optimal threshold we use Non-dominated Sorting Genetic Algorithm II (NSGA-II) which 
is a multi-objective genetic algorithm, proposed by (Deb et al., 2002). In the structure of NSGAII, in addition to genetic operators, crossover and mutation, two specialized multi-objective operators and mechanisms are defined and utilized. These are as follows:

- Non-Dominated Sorting: The population is sorted and partitioned into fronts (F1, F2, etc.), where F1 (first front) indicates the approximated Pareto front.

- Crowding Distance: It is a mechanism of ranking among members of a front, which are dominating or dominated by each other.

We optimize for two objective (i). Correct classification rate $(C C)$, and (ii). Precision of the known intents. The NSGA2 takes threshold values of an intent as the input variable (values ranging from 0.1 to 0.99 ). It then uses prediction scores of samples from the pre-trained base model to perform optimization on the two objective functions, explained in details in Section 3.3.1 to get an optimal threshold for each known intent class. We initialize the population by randomly selecting the values from the range of the threshold variables and then we calculate the two objective values for each entry in the initial population.

Next we perform a non-dominated sorting in the combination of parent and offspring populations and classify them by fronts, i.e. these are sorted in an ascending level of non-domination. Next, we fill new population according to front ranking. If one front is taken partially, crowding-sort is performed.The less dense population are preferred. The offspring population (children) is then created from this new population using crowded tournament selection (It compares by front ranking, if equal then by crowding distance), crossover and mutation operators. The most important solutions (i.e. the best entries) of the population are kept in fronts.

We run the same procedure 1000 times to get a set of optimal thresholds for each known intent class. From this set of thresholds we choose the maximum threshold. This optimal threshold is used to decide upon known and unknown intent samples.

\subsection{Testing}

During testing, when a new sample (unseen class) is encountered it is first fed to the base model (BiLSTM or BERT) to get the corresponding prediction scores. After getting the prediction scores we compare each entries in the prediction scores with the corresponding optimal thresholds and if we find all the entries to be less than the corresponding optimal thresholds we classify that sample as unknown else we classify the sample to the one known intent class for which the prediction score is higher than the corresponding optimal threshold.

\section{Datasets and Experiments}

\subsection{Dataset}

We use three datasets to conduct our experiments. The detailed statistics of the datasets are shown in Table 1. Few example intents from each dataset are shown in Table 2.

\subsubsection{Banking}

This dataset contains fine-grained intents in the banking domain (Casanueva et al., 2020). It contains 77 intents and 13,083 customer service queries.

\subsubsection{Bank-Catridge}

This is a real-world banking domain chat dataset which contains manually updated samples, created through paraphrasing followed by manual verification. This dataset consists of 14 intents in total, consisting of almost 100 samples per intent.

\subsubsection{SNIPS-NLU}

SNIPS-NLU is an English natural language corpus collected in a crowd-sourced fashion to benchmark the performance of voice assistants. It contains 7 intents and almost 2000 samples per intent.

\subsection{Experimental Setups}

We keep $25 \%$ of the overall intent classes in training and validation set as masked while keeping these masked intent samples in the test set as unmasked. To have a fair evaluation on the imbalanced dataset, we randomly select known classes by weighted random sampling without replacement in the training and validation sets. For BERT initialization, we use the weights of the 'bert-baseuncased' model containing 8-layers transformer units. We fine-tune the model on our training sets. We keep learning-rate to $5 \mathrm{e}-5$, the training batch size is 64 and number of training epochs is set to 50. For Bi-LSTM, we set the output dimension as 128 upon which final linear layer is built (according to the number of classes in the dataset). The maximum number of epochs is set to 50 with early 


\begin{tabular}{|l|l|l|}
\hline \multicolumn{1}{|c|}{ Text } & \multicolumn{1}{|c|}{ True Label } & Predicted Label (ADB) \\
\hline Is Visa or Mastercard available? & visa or mastercard & $\begin{array}{l}\text { supported cards } \\
\text { and currencies }\end{array}$ \\
\hline $\begin{array}{l}\text { The app is showing an ATM } \\
\text { withdrawal that I didn't make. }\end{array}$ & $\begin{array}{l}\text { cash withdrawal not } \\
\text { recognized }\end{array}$ & $\begin{array}{l}\text { declined cash } \\
\text { withdrawal }\end{array}$ \\
\hline $\begin{array}{l}\text { I did what you told me earlier } \\
\text { and contacted the seller for a } \\
\text { refund directly, but nothing is } \\
\text { happening! It's been a week and } \\
\begin{array}{l}\text { I still haven't got anything. Please } \\
\text { just give me back my money }\end{array}\end{array}$ & $\begin{array}{l}\text { refund not showing } \\
\text { uo }\end{array}$ & $\begin{array}{l}\text { balance not updated } \\
\text { after cheque or cash } \\
\text { deposit }\end{array}$ \\
\hline
\end{tabular}

Table 3: Samples texts whose intents are mis-classified by the ADB model but are correctly identified by out BERT+NSGA2 model

\begin{tabular}{|c|c|c|c|}
\hline & Banking & Bank_catridge & SNIPS \\
\hline ADB & 66.47 & 72.1 & 69 \\
\hline Bi-LSTM + NT & 22.15 & 64.2 & 33.5 \\
\hline Bi-LSTM + NSGA2 & 35.2 & 82.1 & 49 \\
\hline BERT+NT & 67.2 & 66.1 & 54.3 \\
\hline BERT+NSGA2 & $\mathbf{6 7 . 2}$ & $\mathbf{7 5}$ & $\mathbf{9 0 . 1}$ \\
\hline
\end{tabular}

Table 4: : The F1 scores of detecting unknown intent class samples with $75 \%$ of total intent class as known class on BANKING, Bank Catridge and SNIPS dataset.

stopping. In normal threshold finding we also experiment with different set of thresholds and it was found that the range between(0.1-0.9) gives the best result. In NSGA2 we keep the chromosome size as 1 as we require only 1 optimal threshold per intent class. We experiment with giving different variable ranges as input and find that a range between (0.1-0.9) gives better result. The number of generations is kept to 1000 and with a population size of 100 , num_tour_particips $=2$, tournament_prob $=0.9$, crossover_param $=2$, mutation $\_$param $=5$.

\section{Result and Analysis}

We experiment with different variants of the proposed model as follows: (i). Bi-LSTM + normal-thresholding, (ii). Bi-LSTM + NSGA2 and (iii). BERT + normal-thresholding and (iv). BERT+NSGA2. We also re-implement the ADM model (Shu et al., 2017) and obtain the results on the datasets mentioned in Section 4.Table 4 shows the F1 score of detecting unknown intent class samples with $75 \%$ of total intent class being kept as known on Banking, Bank Catridge and SNIPSNLU dataset. The best results are highlighted in bold. Comparing with the best score of baseline and different variants of our approach we can see that our final model BERT+NSGA2 gives better results than the baseline and the different variants of our proposed model. Comparing with ADB our approach yields $0.7 \%$ improvement on Banking dataset, 3\% improvement on Bank catridge dataset, and $21 \%$ improvement on SNIPS dataset. It can be observed by the results that our BERT+NSGA2 based approach is able to learn tight thresholds to clearly distinguish between known and unknown intent samples. Using Normal thresholding technique where the objective functions are optimised sequentially does not work well as optimizing one objective function can counter the optimization of another objective. This problem is addressed by multi-objective optimization based technique which simultaneously satisfies all the objective functions, finds a set of optimal solutions instead of one optimal solution. Some examples that are correctly classified by the BERT+NSGA2 and not by BERT+NT are shown in Table 5 . We can see that multi-objective optimization plays a vital role in predicting the unknown samples correctly as compared to normal optimization.

Some examples that are correctly classified by the BERT+NSGA2, but not by ADB are shown in Table 3. From the examples we observe that our BERT+NSGA2 gives importance to the words which are there in the unknown intent samples like "refund", "visa", "master_card" and "didn't make" to make the decision between known and unknown intent class. On the other hand, Table 6 shows that there are some samples in the test data which can be miss-classified to one of the similar intent classes. For example the text "I transferred my funds, why did it not go through?" can be miss-classified to "declined_transfer" intent but it actually belongs 


\begin{tabular}{|l|l|l|}
\hline \multicolumn{1}{|c|}{ Text } & \multicolumn{1}{|c|}{ True Label } & \multicolumn{1}{c|}{$\begin{array}{c}\text { Predicted Label } \\
\text { (BERT+NT) }\end{array}$} \\
\hline $\begin{array}{l}\text { What is the number of days I have } \\
\text { to wait for my Europe transfer? }\end{array}$ & $\begin{array}{l}\text { balance not updated } \\
\text { after bank transfer }\end{array}$ & transfer timing \\
\hline $\begin{array}{l}\text { I need to find out why my transfer } \\
\text { didn't get there. }\end{array}$ & declined transfer & $\begin{array}{l}\text { transfer not received } \\
\text { by recipient }\end{array}$ \\
\hline I have a pending cash withdrawal & $\begin{array}{l}\text { balance not updated } \\
\text { after cheque or cash } \\
\text { deposit }\end{array}$ & $\begin{array}{l}\text { pending cash } \\
\text { withdrawal }\end{array}$ \\
\hline $\begin{array}{l}\text { I don't find your services useful } \\
\text { anymore, how do I delete my account? }\end{array}$ & edit personal details & terminate account \\
\hline $\begin{array}{l}\text { Will it cost more money if my } \\
\text { currency needs to be exchanged? }\end{array}$ & exchange via app & exchange charge \\
\hline
\end{tabular}

Table 5: Samples texts whose intents are mis-classified by the BERT + NT model but are correctly identified by out BERT + NSGA2 model

\begin{tabular}{|c|c|c|}
\hline text & true_intent & predicted_intent \\
\hline $\begin{array}{c}\text { Where can I exchange } \\
\text { my money for EUR? }\end{array}$ & fiat_currency_support & exchange_via_app \\
\hline $\begin{array}{c}\text { I transferred money yesterday, } \\
\text { but it still isn't available? }\end{array}$ & pending_transfer & balance_not_updated_after_bank_transfer \\
\hline $\begin{array}{c}\text { I transferred my funds, } \\
\text { why did it not go through? }\end{array}$ & failed_transfer & declined_transfer \\
\hline $\begin{array}{l}\text { My card still hasn't } \\
\text { arrived after 2 weeks. Is it lost? }\end{array}$ & card_arrival & lost_or_stolen_card \\
\hline $\begin{array}{l}\text { How can I fund my top-up } \\
\text { account using my bank account? }\end{array}$ & transfer_into_account & topping_up_by_card \\
\hline
\end{tabular}

Table 6: Samples texts which can be miss-classified to a very similar intent .

to "failed_transfer" intent class. These close intent classes are hard to correctly predict even by humans. Our model is probably learning tighter thresholds because of parallel optimization of objective functions, resulting in better performance in many cases.

\section{Conclusion and Future Work}

In this paper, we have proposed a novel postprocessing method for unknown intent classification. After pre-training the model with labeled samples, our model can automatically learn precise thresholds to separate the known intent from unknown intent sample. Our method does not require data labelled as unknown intent and can recognise open world unknown intents. Our method also does not require model architecture modification of a deep learning intent classifier. Extensive experiments on three benchmark datasets show that our method yields significant improvements over the compared baseline models.

In future we would also like to find categories in unknown intents apart from detecting unknown intents.

\section{Acknowledgement}

The research reported in this paper is supported by the project "Autonomous Goal-Oriented and Knowledge-Driven Neural Conversational Agents", sponsored by Accenture LLP. Prerna acknowledges Accenture LLP for the research internship.

\section{References}

Abhijit Bendale and Terrance E Boult. 2016. Towards open set deep networks. In Proceedings of the IEEE conference on computer vision and pattern recognition, pages 1563-1572.

Inigo Casanueva, Tadas Temčinas, Daniela Gerz, Matthew Henderson, and Ivan Vulić. 2020. Efficient intent detection with dual sentence encoders. arXiv preprint arXiv:2003.04807.

Yun-Nung Chen, Dilek Hakkani-Tür, and Xiaodong He. 2016. Zero-shot learning of intent embeddings for expansion by convolutional deep structured semantic models. In 2016 IEEE International Conference on Acoustics, Speech and Signal Processing (ICASSP), pages 6045-6049. IEEE.

Kalyanmoy Deb, Amrit Pratap, Sameer Agarwal, and TAMT Meyarivan. 2002. A fast and elitist multiobjective genetic algorithm: Nsga-ii. IEEE transactions on evolutionary computation, 6(2):182-197. 
Emmanuel Ferreira, Bassam Jabaian, and Fabrice Lefevre. 2015a. Online adaptative zero-shot learning spoken language understanding using wordembedding. In 2015 IEEE International Conference on Acoustics, Speech and Signal Processing (ICASSP), pages 5321-5325. IEEE.

Emmanuel Ferreira, Bassam Jabaian, and Fabrice Lefevre. 2015b. Zero-shot semantic parser for spoken language understanding. In Sixteenth Annual Conference of the International Speech Communication Association.

Dan Hendrycks and Kevin Gimpel. 2016. A baseline for detecting misclassified and out-of-distribution examples in neural networks. arXiv preprint arXiv:1610.02136.

Lalit P Jain, Walter J Scheirer, and Terrance E Boult. 2014. Multi-class open set recognition using probability of inclusion. In European Conference on Computer Vision, pages 393-409. Springer.

Joo-Kyung Kim and Young-Bum Kim. 2018. Joint learning of domain classification and out-of-domain detection with dynamic class weighting for satisficing false acceptance rates. arXiv preprint arXiv:1807.00072.

Anjishnu Kumar, Pavankumar Reddy Muddireddy, Markus Dreyer, and Björn Hoffmeister. 2017. Zeroshot learning across heterogeneous overlapping domains. In INTERSPEECH, pages 2914-2918.

Shiyu Liang, Yixuan Li, and Rayadurgam Srikant. 2017. Enhancing the reliability of out-ofdistribution image detection in neural networks. arXiv preprint arXiv:1706.02690.

Ting-En Lin and Hua Xu. 2019. Deep unknown intent detection with margin loss. arXiv preprint arXiv:1906.00434.

Han Liu, Xiaotong Zhang, Lu Fan, Xuandi Fu, Qimai Li, Xiao-Ming Wu, and Albert YS Lam. 2019. Reconstructing capsule networks for zero-shot intent classification. In Proceedings of the 2019 Conference on Empirical Methods in Natural Language Processing and the 9th International Joint Conference on Natural Language Processing (EMNLPIJCNLP), pages 4801-4811.

Qingkai Min, Libo Qin, Zhiyang Teng, Xiao Liu, and Yue Zhang. 2020. Dialogue state induction using neural latent variable models. arXiv preprint arXiv:2008.05666.

Martin Mundt, Iuliia Pliushch, Sagnik Majumder, and Visvanathan Ramesh. 2019. Open set recognition through deep neural network uncertainty: Does outof-distribution detection require generative classifiers? In Proceedings of the IEEE/CVF International Conference on Computer Vision Workshops, pages $0-0$.
Eric Nalisnick, Akihiro Matsukawa, Yee Whye Teh, Dilan Gorur, and Balaji Lakshminarayanan. 2018. Do deep generative models know what they don't know? arXiv preprint arXiv:1810.09136.

Peiqing Niu, Zhongfu Chen, Meina Song, et al. 2019. A novel bi-directional interrelated model for joint intent detection and slot filling. arXiv preprint arXiv:1907.00390.

Libo Qin, Wanxiang Che, Yangming Li, Mingheng Ni, and Ting Liu. 2020. Dcr-net: A deep co-interactive relation network for joint dialog act recognition and sentiment classification. In Proceedings of the AAAI Conference on Artificial Intelligence, volume 34, pages 8665-8672.

Libo Qin, Wanxiang Che, Yangming Li, Haoyang Wen, and Ting Liu. 2019. A stack-propagation framework with token-level intent detection for spoken language understanding. arXiv preprint arXiv:1909.02188.

Seonghan Ryu, Sangjun Koo, Hwanjo Yu, and Gary Geunbae Lee. 2018. Out-of-domain detection based on generative adversarial network. In Proceedings of the 2018 Conference on Empirical Methods in Natural Language Processing, pages 714 718.

Sara Sabour, Nicholas Frosst, and Geoffrey E Hinton. 2017. Dynamic routing between capsules. arXiv preprint arXiv:1710.09829.

Lei Shu, $\mathrm{Hu} \mathrm{Xu}$, and Bing Liu. 2017. Doc: Deep open classification of text documents. arXiv preprint arXiv:1709.08716.

Shashank Srivastava, Igor Labutov, and Tom Mitchell. 2018. Zero-shot learning of classifiers from natural language quantification. In Proceedings of the 56th Annual Meeting of the Association for Computational Linguistics (Volume 1: Long Papers), pages 306-316.

Congying Xia, Chenwei Zhang, Xiaohui Yan, Yi Chang, and Philip S Yu. 2018. Zero-shot user intent detection via capsule neural networks. arXiv preprint arXiv:1809.00385.

Yang Yu, Wei-Yang Qu, Nan Li, and Zimin Guo. 2017. Open-category classification by adversarial sample generation. arXiv preprint arXiv:1705.08722.

Chenwei Zhang, Yaliang Li, Nan Du, Wei Fan, and Philip S Yu. 2018. Joint slot filling and intent detection via capsule neural networks. arXiv preprint arXiv:1812.09471.

Hanlei Zhang, Hua Xu, and Ting-En Lin. 2020. Deep open intent classification with adaptive decision boundary. arXiv preprint arXiv:2012.10209. 